\title{
RESEARCH HIGHLIGHT \\ mRNA acetylation: a new addition to the epitranscriptome
}

\author{
P. Cody $\mathrm{He}^{1,2,3}$ and Chuan $\mathrm{He} \mathbb{D}^{1,2,3}$ \\ Cell Research (2019) 29:91-92; https://doi.org/10.1038/s41422-018-0135-2
}

\begin{abstract}
The epitranscriptome, comprising a diverse set of posttranscriptional chemical modifications of $m R N A$, is an important regulator of gene expression. In a recent study, Arango and colleagues identify acetylation of cytidine $\left(\mathrm{ac}^{4} \mathrm{C}\right)$ as a new mRNA modification that increases the stability and translation efficiency of transcripts.
\end{abstract}

mRNA carries a diverse array of post-transcriptional chemical modifications that play important, wide-ranging roles in gene expression. Currently known internal mRNA modifications include methylation of nucleobases and the $2^{\prime}-\mathrm{OH}$ of the ribose sugar, such as $N^{6}$-methyladenosine $\left(\mathrm{m}^{6} \mathrm{~A}\right), N^{1}$-methyladenosine $\left(\mathrm{m}^{1} \mathrm{~A}\right), 5$-methylcytosine $\left(\mathrm{m}^{5} \mathrm{C}\right), 5$-hydroxymethylcytosine $\left(\mathrm{hm}^{5} \mathrm{C}\right)$, 2'-O-methylation $\left(\mathrm{N}_{\mathrm{m}}\right)$, as well as isomerization of uridine to pseudouridine $(\Psi)$ (Fig. 1). Epitranscriptomic modifications alter mRNA metabolism and gene expression by regulating a diverse range of processes, including pre-mRNA nuclear processing, nuclear export, transcript stability and translation. ${ }^{1}$ It is becoming increasingly clear that these modifications play diverse roles in a variety of physiological and disease settings. ${ }^{2}$

Even as the functions of known epitranscriptomic marks are being explored, the list of new mRNA modifications continues to grow. In a recent study published in Cell, Arango and colleagues identify $N^{4}$-acetylcytidine $\left(\mathrm{ac}^{4} \mathrm{C}\right)$ as a new mRNA modification catalyzed by $\mathrm{N}$-acetyltransferase 10 (NAT10), which increases mRNA stability and promotes translation efficiency. ${ }^{3} \mathrm{ac}^{4} \mathrm{C}$ was previously reported to be present at specific sites in eukaryotic serine and leucine tRNAs (tRNA ${ }^{\text {ser/leu }}$ ) and 18S rRNA, with NAT10 identified as the acetyltransferase for $\mathrm{ac}^{4} \mathrm{C}$ in tRNA and rRNA in humans. ${ }^{4}$ However, the prevalence, location and function of $\mathrm{ac}^{4} \mathrm{C}$ on mRNA were unknown. Using antibody-based approaches such as dot blot and immuno-northern blot, as well as liquid chromatography-coupled tandem mass spectrometry (LC-MS/MS), the authors report the presence of $\mathrm{ac}^{4} \mathrm{C}$ on polyadenylated (poly(A)) RNA. Given the role of NAT10 in installing $\mathrm{ac}^{4} \mathrm{C}$ on tRNA and rRNA, they hypothesize that NAT10 may also install $\mathrm{ac}^{4} \mathrm{C}$ on poly(A) RNA. Indeed, they observe that upon knockout of NAT10, $\mathrm{ac}^{4} \mathrm{C}$ levels on both poly(A) RNA and total RNA are significantly depleted.

To map the distribution of $\mathrm{ac}^{4} \mathrm{C}$ sites across the transcriptome, the authors subjected fragmented poly(A) RNA to immunoprecipitation with an anti-ac ${ }^{4} \mathrm{C}$ antibody and utilized next-generation sequencing to measure enrichment of sequences transcriptome wide (acRIP-seq). To identify regions containing $\mathrm{ac}^{4} \mathrm{C}$, they compare acRIP-seq data from wild-type cells to those generated from a $\mathrm{NAT}_{10} \mathrm{O}^{-/}$control cell line and define acetylated regions as those exhibiting higher values of enrichment in the wild type than in the $\mathrm{NAT}_{10} \mathrm{O}^{-1-}$ control. This step was taken to reduce the identification of false-positive peaks that could result from non-specific binding of the antibody to non-acetylated RNA sequences. Using acRIP-seq, they identify $4251 \mathrm{ac}^{4} \mathrm{C}$ peaks throughout the transcriptome. Most $\mathrm{ac}^{4} \mathrm{C}$-marked transcripts contain 1 or 2 peaks. $\mathrm{ac}^{4} \mathrm{C}$ peaks are found throughout the length of mRNAs, but are enriched in the $5^{\prime}$ untranslated region (5' UTR) and coding sequence (CDS).

After mapping the locations of $\mathrm{ac}^{4} \mathrm{C}$ sites, the authors assessed the impact of $\mathrm{ac}^{4} \mathrm{C}$ on gene expression. The authors observe an overall decrease in levels of acetylated transcripts, but not non-acetylated transcripts, in $N A T 10^{-1-}$ cells, suggesting that NAT10-deposited $\mathrm{ac}^{4} \mathrm{C}$ promotes mRNA expression. Analysis of $\mathrm{ac}^{4} \mathrm{C}$ position on the acetylated transcripts indicated that transcripts with CDS or $3^{\prime}$ UTR $\mathrm{ac}^{4} \mathrm{C}$ are preferentially downregulated by loss of NAT10. Supporting a role for $\mathrm{ac}^{4} \mathrm{C}$ in increasing mRNA expression by promoting mRNA stability, transcripts with $C D S \mathrm{ac}^{4} \mathrm{C}$ exhibit an overall decrease in mRNA half-life in the absence of NAT10. The authors next assessed the impact of $\mathrm{ac}^{4} \mathrm{C}$ on translation efficiency by measuring ribosome occupancy on mRNA through sequencing of ribosomeprotected fragments (Ribo-seq). They find that that acetylated mRNAs display elevated translation efficiency compared to nonacetylated mRNAs, and that the ablation of NAT10 decreases translation efficiency of acetylated mRNAs. Given previously reported roles for NAT10 in 18S rRNA processing and maintenance of $40 \mathrm{~S}$ ribosomal subunit levels, as well as its role in installing $\mathrm{ac}^{4} \mathrm{C}$ on tRNA $\mathrm{Aer}^{\text {seu }}$, the observed effects of NAT10 depletion on translation could possibly be mediated by functions of $\mathrm{ac}^{4} \mathrm{C}$ on tRNA or rRNA. ${ }^{4,5}$ However, in NAT10 ${ }^{-/-}$ cells, the authors report normal polysome profiles and 18S rRNA processing and do not detect increased ribosome stalling at serine or leucine codons in their Ribo-seq data, suggesting that the effects of NAT10 depletion on tRNA or rRNA might not drive the observed effect on translation.

Mechanistically, the authors propose that $\mathrm{ac}^{4} \mathrm{C}$ on mRNA promotes translation by facilitating tRNA decoding when present in the third nucleotide of codons, known as the wobble position. They find that codons containing cytidine in the wobble position are enriched within $\mathrm{ac}^{4} \mathrm{C}$ peaks, but not codons containing cytidine in the first or second positions. They further identify a CXX repeat motif enriched within $\mathrm{ac}^{4} \mathrm{C}$ peaks in which the obligate cytidine consistently falls within the wobble position of the reading frame. Notably, it has previously been shown that $\mathrm{ac}^{4} \mathrm{C}$ in a bacterial tRNA ${ }^{\text {eMet }}$ anticodon wobble site facilitates translational fidelity by tuning codon-anticodon affinity. ${ }^{6}$ The authors test the functional significance of wobble site $\mathrm{ac}^{4} \mathrm{C}$ in translation using both in vitro translation assays and transfection of in vitro transcribed reporter mRNA. They find that incorporation of $\mathrm{ac}^{4} \mathrm{C}$ into reporter transcripts by in vitro transcription markedly

\footnotetext{
${ }^{1}$ Department of Chemistry, Department of Biochemistry and Molecular Biology, Institute for Biophysical Dynamics, The University of Chicago, Chicago, IL 60637, USA; ${ }^{2}$ Committee on Immunology, The University of Chicago, Chicago, IL 60637, USA and ${ }^{3}$ Howard Hughes Medical Institute, The University of Chicago, Chicago, IL 60637, USA

Correspondence: Chuan He (chuanhe@uchicago.edu)
}

Published online: 10 January 2019 


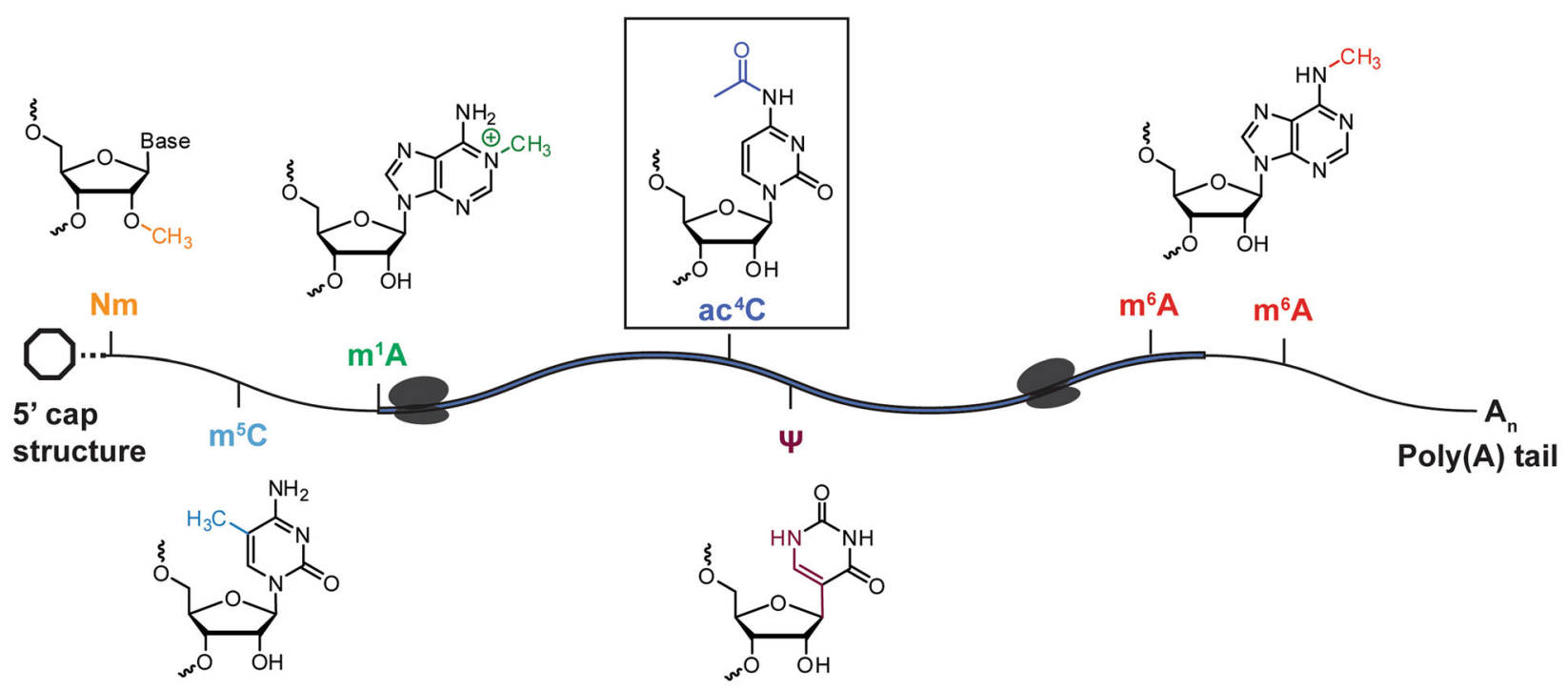

Fig. 1 A schematic representation of common chemical modifications in eukaryotic mRNA transcripts. Several of these modifications map preferentially to the mRNA cap structure, $5^{\prime}$ or $3^{\prime}$ untranslated regions, or the coding region (bold) of the transcript. $N^{4}$-acetylcytidine (ac ${ }^{4} \mathrm{C}$ ) (boxed) is a newly reported addition to the known chemical modifications on mRNA

increases translation, but that this effect is muted when synonymous mutations are introduced to remove cytidines from wobble positions, supporting a specific role for wobble position $\mathrm{ac}^{4} \mathrm{C}$ in promoting translation.

Arango et al. establish acetylation as a new class of mRNA modification, expanding the epitranscriptome beyond the known classes of methylation and isomerization. The authors report an intriguing role for wobble position $\mathrm{ac}^{4} \mathrm{C}$ in translation, with interesting parallels to the well-established paradigm of tRNA modifications at the anticodon wobble site impacting codon-anticodon affinity in order to tune translation. This report, along with other recent studies reporting the presence of $m^{1} A$, $\mathrm{m}^{5} \mathrm{C}$ and its derivatives, and $\mathrm{Nm}$ in mRNA, represent a flurry of recent work that have expanded the known repertoire of mRNA modifications and established the presence of a diverse array of chemical modifications on mRNA. ${ }^{7-10}$ New modes of mRNA modification may well continue to emerge in the future. Further work to understand the functions of these modifications, the pathways and mechanisms that regulate their presence on specific transcripts, as well as their functions in various physiological and disease contexts will be integral for a more complete understanding of the roles of the epitranscriptome in gene expression.

\section{ADDITIONAL INFORMATION}

Competing interests: The authors declare no competing interests.

Publisher's note: Springer Nature remains neutral with regard to jurisdictional claims in published maps and institutional affiliations.

\section{REFERENCES}

1. Roundtree, I. A. et al. Cell 169, 1187-1200 (2017).

2. Frye, M. et al. Science 361, 1346-1349 (2018).

3. Arango, D. et al. Cell 2018. https://doi.org/10.1016/j.cell.2018.10.030

4. Sharma, S. et al. Nucleic Acids Res. 43, 2242-2258 (2015).

5. Ito, S. et al. J. Biol. Chem. 289, 35724-35730 (2014).

6. Taniguchi, T. et al. Nat. Chem. Biol. 14, 1010-1020 (2018).

7. Li, X. et al. Mol. Cell 68, 993-1005 (2017).

8. Yang, X. et al. Cell Res. 27, 606-625 (2017).

9. Delatte, B. et al. Science 351, 282-285 (2016).

10. Dai, Q. et al. Nat. Methods 14, 695-698 (2017). 